\title{
Interaktionen ZWischen Mensch Und Karst Ein integratives Funktionsmodell und seine praktische Anwendung am Beispiel Tanneben, Steiermark
}

\author{
Christian BAUER, Graz*
}

mit 10 Abb. und 4 Tab. im Text

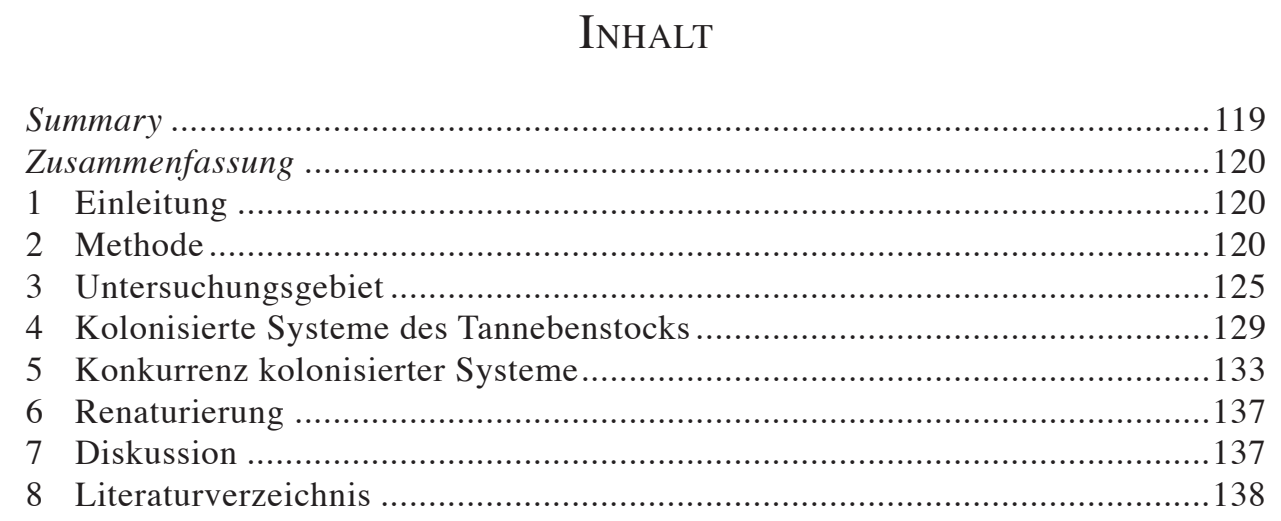

\section{Summary}

Interface between society and karst. An integrative functional model and its practical application by the example of Tanneben, Styria

The paper focuses on the interface between society and nature on karst by using the example of Tanneben, Styria. Strong human agency impacts on earth's surface increasingly raise questions for setting up modern theoretical concepts to analyse those influences. Theory and applicability of the conceptual model of colonisation of nature are discussed by using a socially-ecologically based integrative functional model on karst, in the case of Tanneben.

Mag. Christian BAUER, Institut für Geographie und Raumforschung, Karl-Franzens-Universität Graz, Heinrichstraße 36,A-8010 Graz; e-mail: bauerchr@edu.uni-graz.at,http://www.kfunigraz.ac.at/wwwgeo 


\section{Zusammenfassung}

Der Beitrag thematisiert Mensch-Umwelt-Beziehungen im Bereich des geomorphologischen Landschaftstyps Karst am Beispiel des Tannebenstocks, Steiermark. Die enormen Auswirkungen menschlichen Handelns auf das System Erdoberfläche werfen vermehrt Fragen betreffend geeigneter theoretischer Konzepte zur Erfassung dieser Einflussnahmen auf. Der Beitrag diskutiert Theorie und praktische Implementierung des Konzepts der Kolonisierung von Natur mittels des Mensch-Karst-Interaktionsmodells anhand einer Fallstudie (Tannebenstock).

\section{Einleitung}

In Anlehnung an das methodologisch-theoretische Konzept der Sozialen Ökologie untersucht der Beitrag die praktische Implementierung eines Mensch-UmweltInteraktionsmodells am Beispiel des geomorphologischen Landschaftstyps Karst. Das entworfene Modell versteht sich als epistemologisches Werkzeug einer zeitgemäßen integrativen geographischen Forschung (Stichwort Dritte Säule), dessen bisherige Ergebnisse neue Facetten der Mensch-Umwelt-Interaktionen zeigen: die Positionierung einer ,Umwelt ${ }^{6}$ - im konkreten Fall eines geomorphologischen Landschaftstyps - als Gegenstand eines Interessenskonfliktes, an dem der Mensch nicht nur als statischstörender, sondern als agierender und reagierender Agent des Systems teilnimmt. Der ausgezeichnete (über 120 Jahre zurückreichende) Forschungsstand und die Vielzahl an Mensch-Karst-Interaktionen weisen den Tannebenstock als geeignetes Untersuchungsgebiet aus.

\section{Methode}

Der Kernpunkt sozialökologischer Forschungen ist die Annahme eines von Wechselwirkungen geprägten, gekoppelten Systems von Gesellschaft und Natur (FISCHERKowALSKI \& HABERL 1997a). Die theoretischen Grundlagen dieser Annahme sind Gegenstand methodologischer, fachpolitischer Diskussionen, deren ganze Bandbreite in diesem Beitrag nicht erörtert werden kann. Die in Bezug auf das in diesem Artikel verwendete Mensch-Karst-Interaktionsmodell (folgend abgekürzt als M-K-I) wichtigsten sozialökologischen Erkenntnismodi seien in aller Kürze zusammengefasst:

- ein nicht dichotomes Verständnis von Kultur (K) und Natur (N) mit Überschneidungsbereich (hybrides System: grauer Bereich in Abb. 1): Forschungsgegenstände können sowohl $\mathrm{K}$ als auch $\mathrm{N}$ angehören;

- die Konzeption einer über den Akteur Population (P) sicher gestellten, materiellphysisch agierenden Gesellschaft: hierin liegt ein großer Bruch mit den Sozial- 


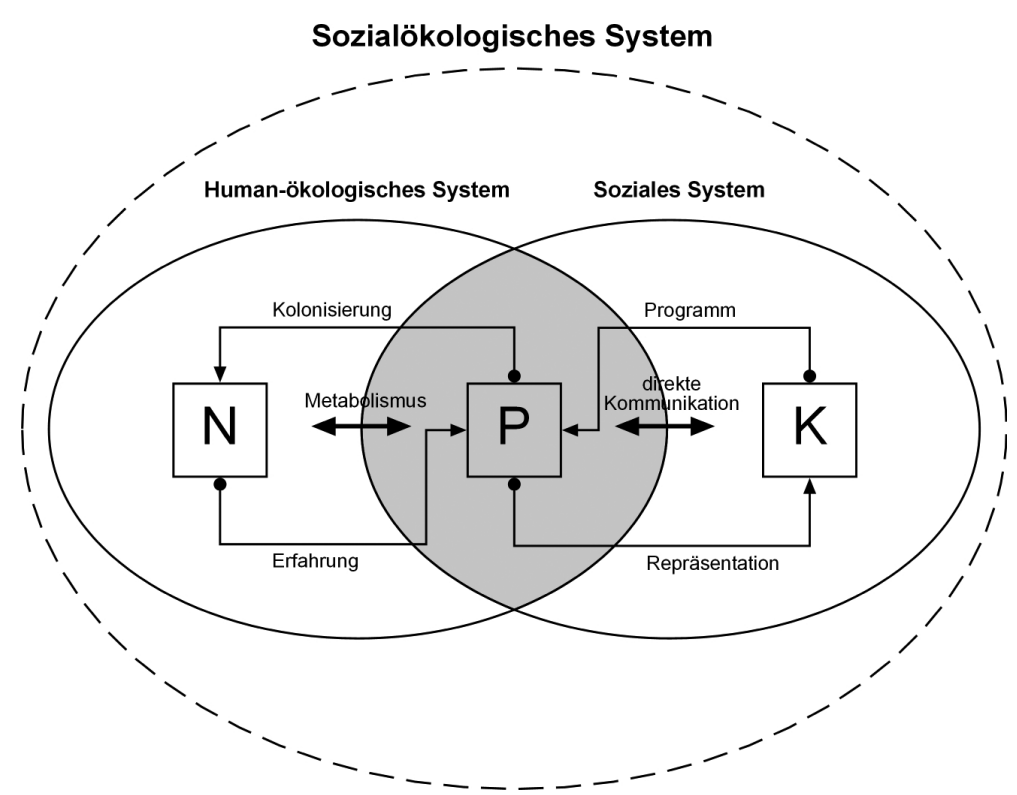

Abb. 1: Sozialökologisches Interaktionsmodell nach FisCHER-KOWALSKI \& ERB (2006); veränderte, eigene Darstellung

Zum Andocken an bestehende Forschungsmethoden empfiehlt SIEFERLE (1997) die Ausdifferenzierung in ein Human-ökologisches System und ein Soziales System. Für die Details sei auf die Publikationen rund um Fischer-KowaLSKI verwiesen.

wissenschaften, die Gesellschaft üblicherweise als rein kommunikatives System definieren;

- das Konzept der Kolonisierung als zielgerichtete Intervention in natürliche Systeme, deren Aufrechterhaltung eine ständige Investition von Arbeit benötigt;

- das Konzept der Renaturierung bei Erliegen der Kolonisierungstätigkeiten: Renaturierung bedeutet ein Erstarken naturaler Prozesse, aber keine Rückkehr in das natürliche System;

- das Konzept des gesellschaftlichen Metabolismus, wonach Gesellschaften - Organismen gleich - zur Aufrechterhaltung einen ständigen Durchsatz von Materie/ Energie benötigen.

Ausgangspunkt des M-K-I (vgl. Tab. 1) ist die Überlegung, dass der Mensch sowohl im Bezugssystem der Raumnutzung (dem Äquivalent des Human-ökologischen Systems) als auch der Raumbewertung (dem Äquivalent des Sozialen Systems) in Interaktion mit definierten Karstaspekten (z.B. Hohlform) des geomorphologischen Landschaftstyps Karst tritt. Diese Interaktionen werden über das Prinzip der Kolonisierung analysiert. Bei einem Verständnis von Kolonisierung als zielgerichtete, anthropogene Intervention natürlicher Systeme ergibt sich logischerweise die Frage nach deren Zweck. Im M-K-I wird der Zweck über Funktionen (klassifiziert in 


\begin{tabular}{|c|c|c|c|c|}
\hline $\begin{array}{l}\text { Bezugs- } \\
\text { system }\end{array}$ & Karstaspekt & Schlüsselfunktion & Unterfunktion & Rückwirkungen \\
\hline \multirow{10}{*}{ 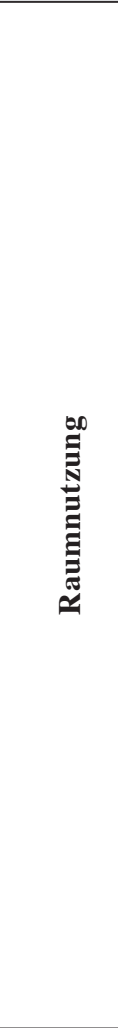 } & \multirow{3}{*}{ Hohlform } & $\begin{array}{l}\text { Orte verbergungs- } \\
\text { würdiger Aktivitäten }\end{array}$ & $\begin{array}{l}\text { Abfallentsorgung } \\
\text { Kultische Aktivitäten } \\
\text { Produktionsstätten }\end{array}$ & \multirow{13}{*}{ 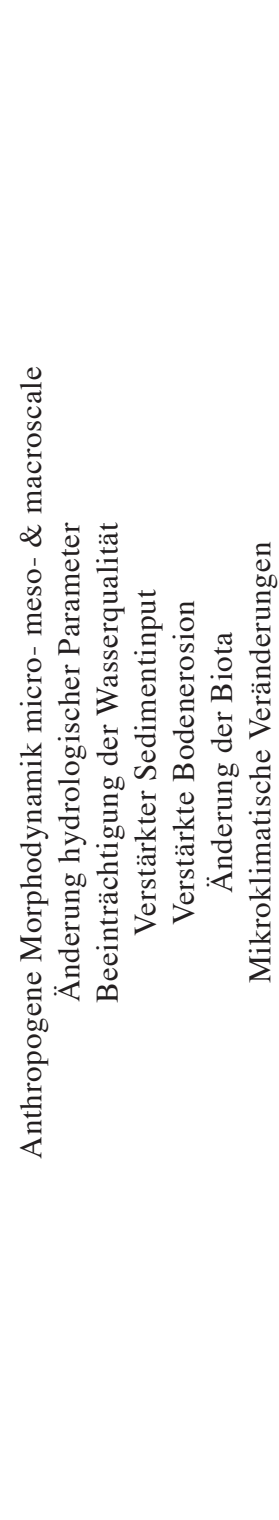 } \\
\hline & & Schutzfunktion & $\begin{array}{l}\text { Schutz von Eigentum } \\
\text { Witterungsschutz } \\
\text { Fortifikationen }\end{array}$ & \\
\hline & & $\begin{array}{l}\text { Konstantes } \\
\text { Temperaturniveau }\end{array}$ & Kühlfunktion & \\
\hline & Wasser & $\begin{array}{l}\text { Trinkwasser } \\
\text { Heilwasser } \\
\text { Energie } \\
\text { Transportmedium }\end{array}$ & & \\
\hline & $\begin{array}{l}\text { Formen- } \\
\text { vielfalt }\end{array}$ & $\begin{array}{l}\text { Erholung } \\
\text { Touristische Nutzung }\end{array}$ & & \\
\hline & Luft & $\begin{array}{l}\text { bioklimatische } \\
\text { Wirkung }\end{array}$ & & \\
\hline & Sedimente & $\begin{array}{l}\text { Düngemittel } \\
\text { Volksmedizin } \\
\text { Ästhetische Funktion }\end{array}$ & & \\
\hline & & Landwirtschaft & $\begin{array}{l}\text { Forstwirtschaft } \\
\text { Almwirtschaft }\end{array}$ & \\
\hline & Fläche & Transport & & \\
\hline & & Industrie & Bergbau & \\
\hline \multirow{3}{*}{ 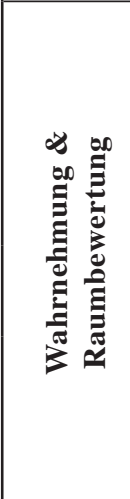 } & \multirow{3}{*}{$\begin{array}{l}\text { Landschaft- } \\
\text { liche Einzig- } \\
\text { artigkeit; } \\
\text { karstspezifi- } \\
\text { sche Raum- } \\
\text { ausstattung }\end{array}$} & $\begin{array}{l}\text { Objekt der } \\
\text { Wahrnehmung; } \\
\text { Identitätsstiftung }\end{array}$ & $\begin{array}{l}\text { Onomastik } \\
\text { Inschriften } \\
\text { Mythen, Märchen, } \\
\text { Sagen } \\
\text { Literatur } \\
\text { Bildende Kunst } \\
\end{array}$ & \\
\hline & & $\begin{array}{l}\text { Karstformen als } \\
\text { Herausforderung }\end{array}$ & & \\
\hline & & $\begin{array}{l}\text { Wissenschaftlicher } \\
\text { Wert }\end{array}$ & $\begin{array}{l}\text { Kulturhistorisches } \\
\text { Archiv } \\
\text { Klimaindikatoren } \\
\text { Umweltdidaktik }\end{array}$ & \\
\hline
\end{tabular}

Tab. 1: M-K-I. Stark vereinfachte tabellarische Darstellung. Aufgrund des Zeitschriftenformates ist eine grafische Komplettdarstellung des Modells nicht möglich. 
a

Kolonisiertes System

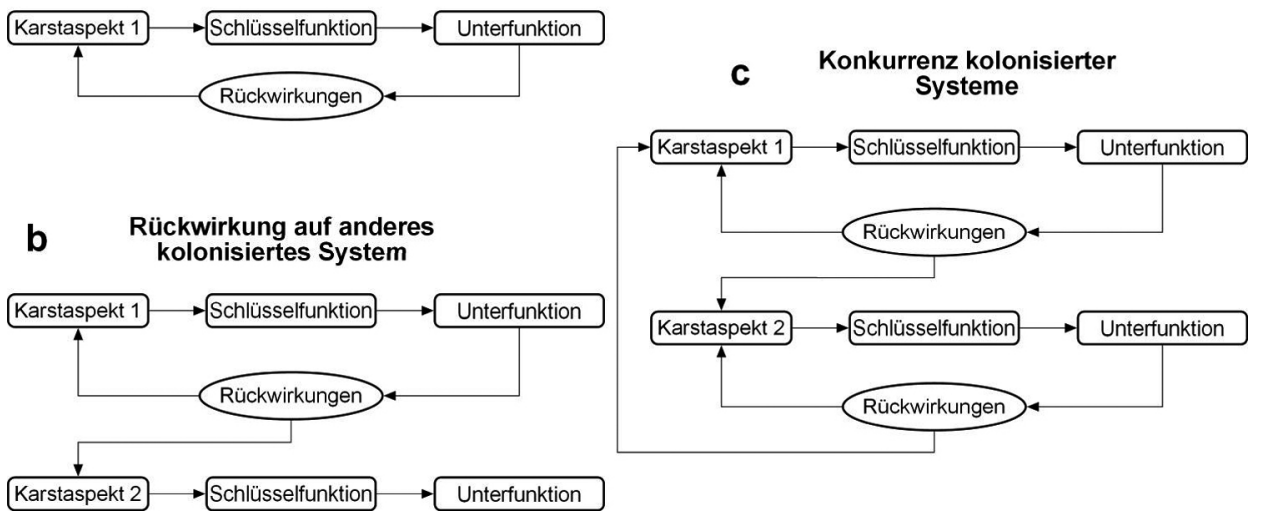

Abb. 2: Prinzip kolonisierter Systeme im M-K-I

Schlüsselfunktionen und gegebenenfalls Unterfunktionen) definiert. Die eigentliche Kolonisierungstätigkeit (investierte Arbeit) impliziert intendierte und nicht-intendierte Rückwirkungen (z.B. Änderung hydrologischer Parameter) auf den zu kolonisierenden Karstaspekt (vgl. Abb. 2a). Die Wahrscheinlichkeit, dass diese Rückwirkungen im sehr sensitiv reagierenden geomorphologischen Landschaftstyp Karst andere Karstaspekte - damit auch andere Funktionen - beeinflussen, ist sehr groß (vgl. Abb. 2b). Die Erhaltung oder Aufrechterhaltung der einzelnen Funktionen resultiert schließlich in einem untereinander rückgekoppelten, konkurrierenden System kolonisierter Systeme (vgl. Abb. 2c). Diese Konfliktsituationen werden in das Soziale System transferiert und über Regelmechanismen (z.B. Naturschutz) ausgetragen.

Besonders im Karst besteht eine große Herausforderung in der Identifizierung prozessualer Wirkungszusammenhänge (Rückwirkungen) zwischen kolonisierenden Eingriffen und Karstaspekten (vgl. Abb. 3).

Die Datengrundlage des Modells (Kernpunkt des Dissertationsvorhabens des Autors) bildete eine beinahe lückenlose Aufarbeitung der karstrelevanten Literatur zur Steiermark an verschiedenen Institutionen. Der zusammengetragene Datensatz umfasst mehr als 640 Einträge unterschiedlicher Dokumenttypen, meist jedoch Aufsätze aus Zeitschriften und Sammelbänden. Ergänzt wurde diese intensive Recherchetätigkeit durch eine ausgedehnte Geländearbeit inklusive Fotodokumentation. Die kartographische Umsetzung mittels eines GIS (ArcView 9.2) ermöglicht nicht nur weiterführende Analysen, sondern auch eine gute Visualisierung von karstmorphologischen und -hydrologischen Charakteristika und deren Interaktionen mit dem Menschen. 


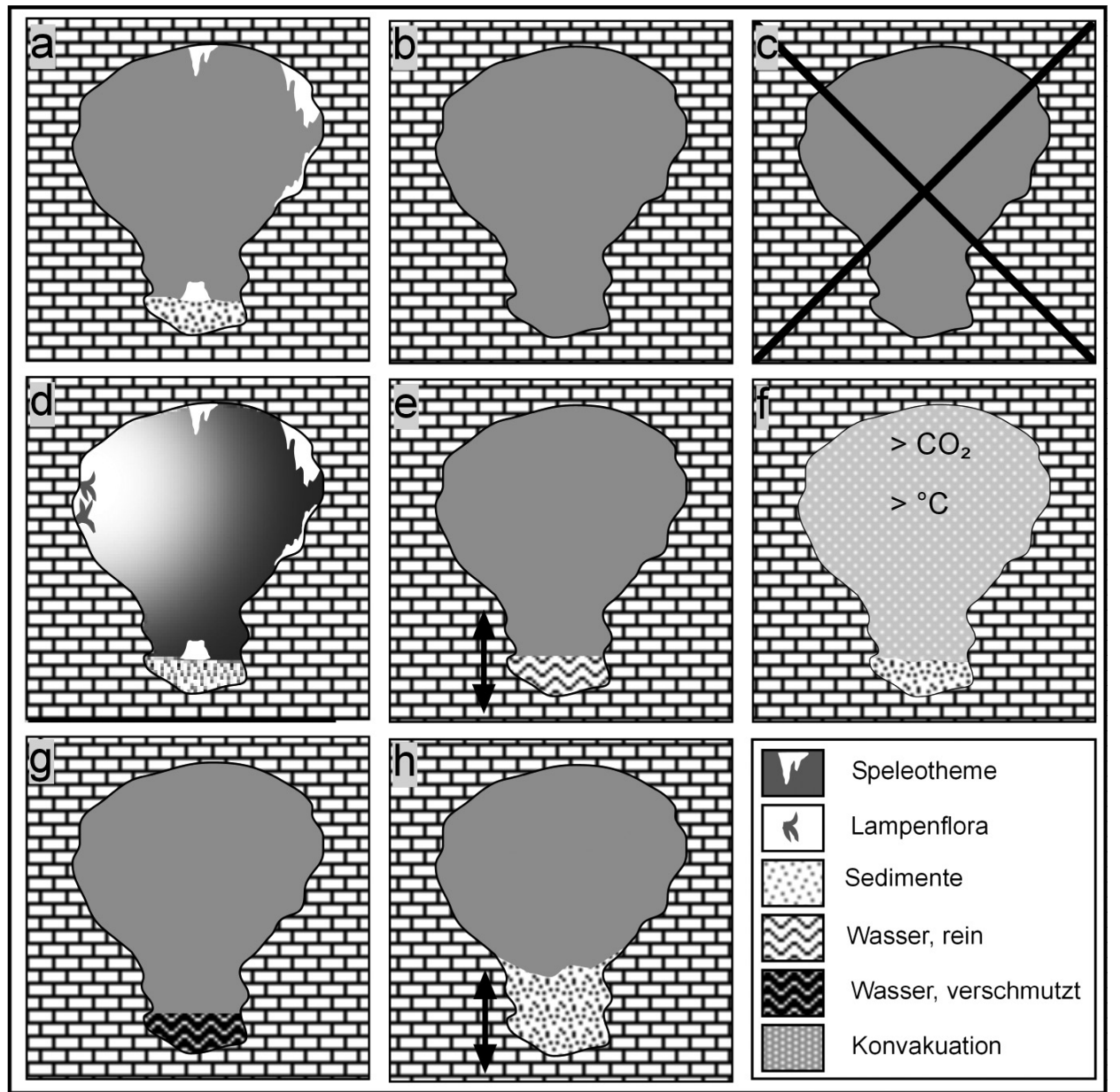

Abb. 3: Rückwirkende Prozesse im M-K-I am Beispiel des Karstaspekts Hohlform Höhlenpassage phreatischer und vadoser Genese (Schlüssellochprofil)

3a: Anthropogene Morphodynamik microscale: Veränderungen am festen Höhleninhalt (Speläotheme, Eis);

3b: Anthropogene Morphodynamik mesoscale: Veränderungen am Evakuationsraum (Grenzfläche zum Muttergestein);

3c: Anthropogene Morphodynamik macroscale: Zerstörung der Passage (Höhle);

3d: Änderungen an Biota: Eintrag von Fremdarten (z.B. Lampenflora);

3e: Änderung hydrologischer Parameter: Ab- und Zufuhr von Wasser;

3f: Mikroklimatische Veränderungen in der Konvakuation: erhöhte Temperatur / $\mathrm{CO}_{2}$-Konzentration durch Schauhöhlenbetrieb;

3g: Änderung der Wasserqualität: Eintrag von Schadstoffen;

3h: Verstärkter Sedimentinput: durch Rodungsprozesse an der Oberfläche. 


\section{Untersuchungsgebiet}

Der Tannebenstock (oder auch das Tannebenmassiv oder die Tanneben) wird vielfach als Herzstück des von Воск (1913) postulierten Mittelsteirischen Karstes bezeichnet. Dieses rund $2.000 \mathrm{~km}^{2}$ große, überwiegend aus paläozoischen (Devon) Karbonaten (eine Ausnahme bilden die neogenen Karbonate im Raum Wildon, Aflenz/Retznei) aufgebaute Karstgebiet besitzt Mittelgebirgscharakter und ist aufgrund der beinahe durchgehend geschlossenen Vegetationsbedeckung (diese wird nur in Bereichen extremer Reliefenergie unterbrochen) als Grünkarst zu klassifizieren. Der Beginn der Verkarstung wird in das jüngere Miozän datiert (WINKLER-HERMAden 1957), wobei einzelne, u.a. von PASCHINGER (1965) kartierte, reliktische Karstformen (Kuppenkarst) älterer Genese (mittleres Miozän) entstammen.

Die Morphogenese des Mittelsteirischen Karstes ist charakterisiert durch etappenweises Emporheben, beginnend im Pannonium, isostatische Ausgleichsbewegungen und Rücksenkungen (MAURIN 1994), die in eine Gliederung einzelner Schollen und zu deren Verschiebung oder Verbiegung führte. Der geologisch überwiegend aus Schöcklkalk bestehende Tannebenstock entspricht einer solchen Teilscholle und stellt damit ein anschauliches Ergebnis dieser Prozesse dar (MAURIN 1952).

Ein weiterer morphologisch bedeutender Faktor besteht in der parallel stattfindenden Tiefenerosion: So ist das geologisch idente Karstgebiet des Kugelsteins und der Feistritzer Wand durch die Tiefenerosion der Mur vom Tannebenmassiv isoliert worden. Gleiches gilt für das durch den Badlbach oder Mühlbach nördlich des Badlgrabens gelegene isolierte Himmelreich. Diese hydrographischen Tiefenlinien grenzen das Untersuchungsgebiet (vgl. Abb. 5) gegen W und N (bis Schönegg) ab. Vervollständigt wird die Abgrenzung durch eine Linie Schönegg - Neudorf - Dreihöfen - Anger bis knapp oberhalb des Rötschbach-Ursprungs, dessen Verlauf zugleich die Südgrenze des Untersuchungsgebietes ist. Grundlage dieser Grenzziehung - die über die eigentliche Kalkscholle des Tannebenstocks hinaus geht - bildet der Höhlenkataster des Österreichischen Höhlenverzeichnisses (im Folgenden abgekürzt als ÖHV) des Verbands Österreichischer Höhlenforscher (STUMmER \& Plan 2002).

Das Heranziehen dieses wissenschaftlich bereits etablierten Systems ist nicht nur pragmatisch, sondern bietet auch gute Vergleichsmöglichkeiten. Alle im Text erwähnten Höhlen sind mit dem Kennziffernsystem des ÖHV versehen. Das Wechselspiel aus orogenen Hebungen und tektonischer Stabilität resultiert in einer Ausbildung von Verebnungssystemen (vgl. Tab. 2). Karstmorphologisch bedeutsam können tektonische Ruhephasen vor allem im Hinblick auf die Speläogenese sein: so ist (im Allgemeinen) auch mit einem stabilen Vorflut- oder Karstwasserniveau und damit mit Etagenausbildung zu rechnen. Ein erneutes Erstarken orogener Faktoren resultiert in einer Tieferlegung des Vorflut- oder Karstwasserniveaus. Denselben Effekt bewirkt auch ein erosionsbedingtes Einschneiden des Vorfluters (am Beispiel Tannebenstock der Mur).

Indikatoren für die intensive Verkarstung des Untersuchungsgebietes sind sowohl die vollständig ausgeprägte karsthydrologische Entwässerung (kein Oberflächenabfluss im Bereich des Tannebenmassivs, starke Karstquellen) als auch die Vielzahl von Karsthöhlen. Das ÖHV listet 232 Höhlen auf 47,1 km² Fläche auf (Stand 2002). Dies 


\begin{tabular}{|l|c|l|l|}
\hline Niveauname & Höhen [m] & Befunde & Beispiel Lokalität \\
\hline Trahütten-Niveau & $\sim 900$ & Keine & \\
\hline Hochstradner-Niveau & $760-720$ & Höhlen, Dolinen & W \& S Tannebenstock \\
\hline Stadelberg-Niveau & $640-680$ & Roterden, Schotter & Angerleitenkogel \\
\hline
\end{tabular}

Tab. 2: Verebnungssysteme im Bereich Tannebenstock. Da im gesamten Untersuchungsgebiet nur in Ausnahmenfällen das 900 m-Niveau überschritten wird, finden sich kaum Befunde für das Trahüttenniveau.

entspricht einer Höhlendichte von 4,93 Höhlen $/ \mathrm{km}^{2}$ - ein Wert, der in der Steiermark nur im Bereich des Toten Gebirges vom Katastergebiet Loser - Augsteck [1623] mit 7,69 Höhlen $/ \mathrm{km}^{2}$ übertroffen wird.

Trotz über 100 Jahre währender speläologischer Forschungen (vgl. WURMBRAND 1871) überrascht das Untersuchungsgebiet immer wieder mit Neuentdeckungen. So gelang zum Beispiel 2001 die Entdeckung der Großhöhle Moosschacht [2836/237] (KUSCH 2004). Neben der bereits erwähnten vollständigen unterirdischen Entwässerung der Kalkscholle des Tannebenmassivs (mit einem Einzugsgebiet von $\sim 8,5 \mathrm{~km}^{2}$ ) ist vor allem das allochthone Gewässer des Lurbaches von karsthydrologischer Bedeutung.

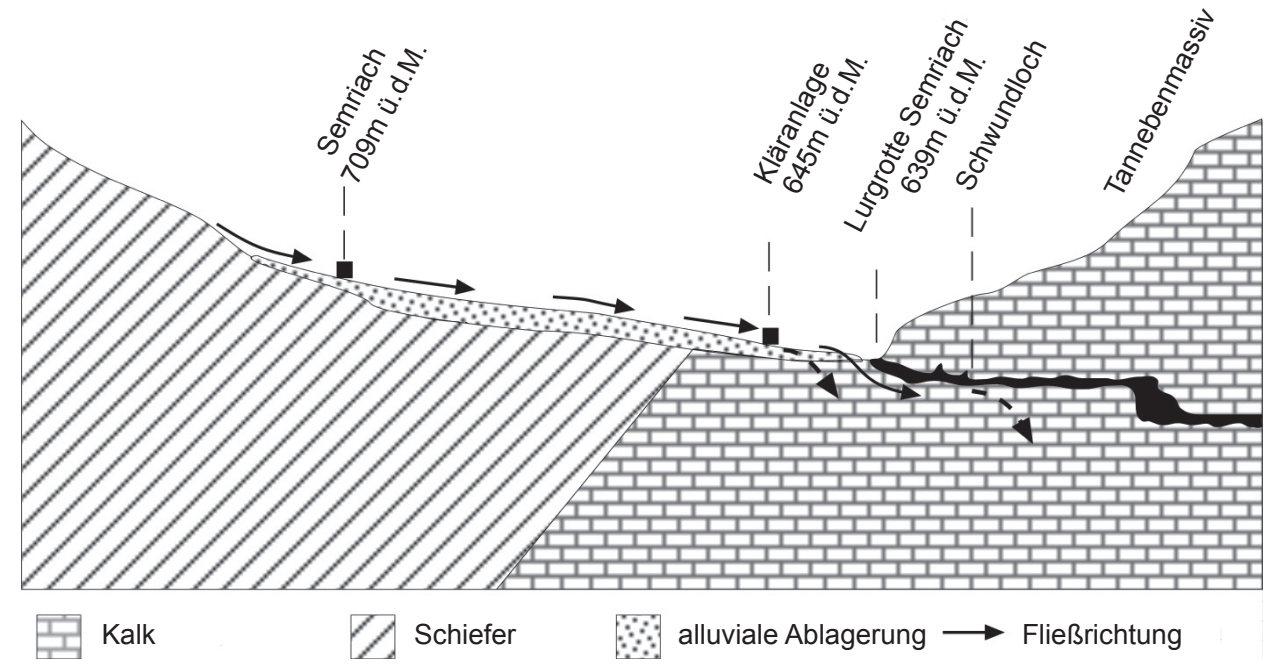

Abb. 4: Schematisches Profil des Border Polje im Raum Semriach

Das 18,3 km² große Gebiet wird zur Gänze durch den Lurbach entwässert. Ab dem Kontakt mit dem unterlagernden Schöcklkalk verliert der Bach fortwährend Wasser. BATSCHE et al. (1967) geben hierfür einen Wert von 66,5 1/s (= 28\%) für NQ an. Schließlich verschwindet der Bach (bei NQ \& MQ) vollständig in der sog. Lurbachschwinde nach ca. $200 \mathrm{~m}$ Verlauf als Höhlengerinne und tritt als Hammerbach [2836/34] bei Peggau wieder zu Tage. Nur bei HQ (vgl. Abb. 5) erfolgt eine Entwässerung in den ansonsten trockenen (Führungs-) Teil der Lurhöhle Semriach [2836/1a] (Bauer \& Kellerer-Pirklbauer 2009). 
Dieser entwässert das $18,3 \mathrm{~km}^{2}$ große Border Polje (Klassifizierung von FORD \& Williams 2007) von Semriach (vgl. Abb. 4).

Ein Ponor im Bereich Neudorf (Eisgrube [2836/4]) und ein weiterer N von Pöllau - die Katzenbachschwinde (vgl. Abb. 5) - sind (neben weiteren kleineren Schwinden) die wichtigsten Gerinne. Dem gegenüber stehen zwei starke Karstquellen im W, am Fuße der Peggauer Wand: der Schmelzbach (das Höhlengerinne der Lurgrotte Peggau [2836/1b] und der Hammerbach (vgl. Tab. 3). Bei einer Schüttung des Hammerbaches von $>200$ 1/s erfolgte ein Überlauf in dem bei NQ völlig voneinander isolierten Schmelzbach-Aquifer (BenischKe \& Harum 1994, S. 147).

\begin{tabular}{|l|c|c|c|l|}
\hline Gewässer & $\begin{array}{c}\text { NQ } \\
{[\mathbf{l} / \mathbf{s}]}\end{array}$ & $\begin{array}{c}\text { MQ } \\
{[\mathbf{l} / \mathbf{s}]}\end{array}$ & $\begin{array}{c}\text { HQ } \\
{[\mathbf{l} / \mathbf{s}]}\end{array}$ & Datenreihe \\
\hline Lurbach & 4 & 141 & 15.300 & $1965-1973 ; 1987-1989$ \\
\hline Hammerbach & 33 & 193 & 2.000 & $1965-1975 ; 1983-1989$ \\
\hline Schmelzbach & 15 & 79 & 11.400 & $1965-1970 ; 1983-1989$ \\
\hline
\end{tabular}

Tab. 3: Hydrologische Daten bedeutender Gerinne im Untersuchungsgebiet nach BenischKe \& HARUM (1994)

Die rezenten Austrittslagen der beiden großen Karstquellen (Schmelzbach und Hammerbach) im W des Tannebenmassivs - sie markieren auch das WürmterrassenNiveau - dürfen nicht darüber hinwegtäuschen, dass das eigentliche Karstniveau teilweise unter dem heutigen Austrittsniveau liegt. Inundation eines bereits entwickelten Karstsystems als Folge postglazialer Aufschüttungen der Mur bewirkte Höher- oder Rückverlegen des Wassers im Karstsystem (MAURIN 1961, S. 179).

Dolinen sind im Untersuchungsgebiet, aber auch im gesamten Mittelsteirischen Karst die dominierende Exokarstform. Bereits 1940 wurde von VORMAIR eine detaillierte Untersuchung hinsichtlich der Größe und Verteilung der Dolinen im Mittelsteirischen Karst vorgenommen, die letztendlich in eine Klassifizierung von Dolinenbezirken mündete. Dabei weist das Untersuchungsgebiet Tanneben (inklusive Hochtrötsch) eine sehr hohe Anzahl an Dolinen mit einem Durchmesser von 30 bis 100 m auf. Die von Vormair postulierte Korrelation von Dolinenbezirken mit dem Verebnungssystem des Hochstradner Niveaus - 57\% aller Großformen sind auf dieses Niveau im Grazer Bergland verteilt - soll an dieser Stelle nicht verschwiegen werden. Allerdings darf diese Korrelation nicht als einzige Ursache für diese Verteilung gewertet werden. Als wichtige Gründe der Dolinengenese auf diesem Niveau müssen neben den orographischen Ruhephasen (Zeit) vor allem klimatische Einflüsse gelten (vgl. Maurin 1961). Morphogenetisch sind im Untersuchungsgebiet Lösungsdolinen und Einsturzdolinen zu klassifizieren.

Unter den Kleinformen des Exokarstes sind vor allem Karren zu nennen. Deren Morphogenese ist durch das Vorhandensein einer geschlossenen Vegetationsbedeckung und dem daraus resultierenden erhöhten biogenen $\mathrm{CO}_{2}$-Angebot geprägt: abgerundete Trennstege zwischen den einzelnen Karren (demnach sinngemäß als Rundkarren bezeichnet) sind die Folge dieser intensiven Korrosionsleistung. 


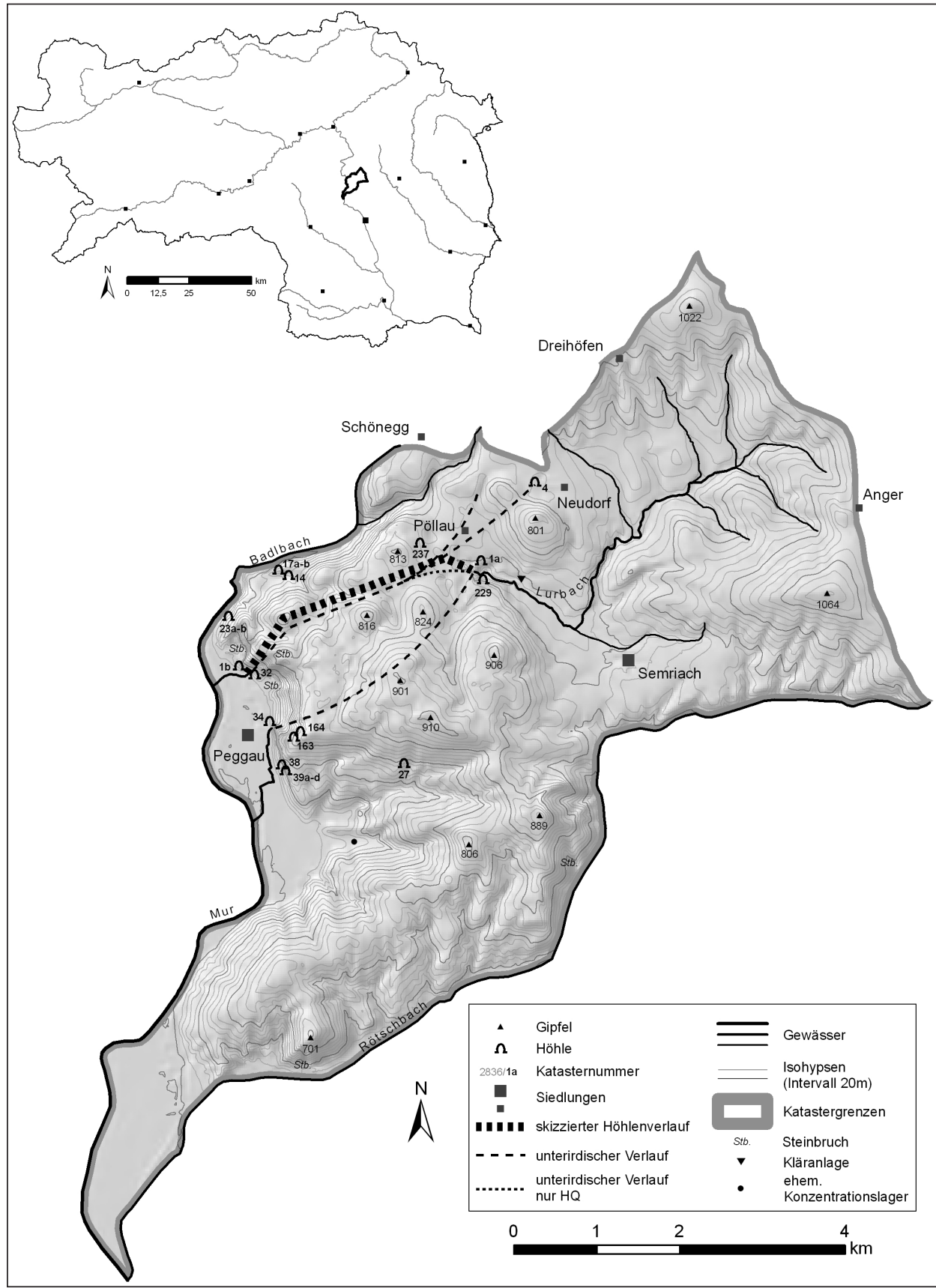

Abb. 5: Untersuchungsgebiet Tanneben, Kat.-Nr. 2836 des Österreichischen Höhlenkatasters. Die Gesamtfläche des Gebietes beträgt 47,1 km². Aus Gründen der Übersichtlichkeit des Kartenbildes wurden verortete Höhlen nur mit der fortlaufenden Nummer beschriftet. 


\section{Kolonisierte Systeme des Tannebenstocks}

Die funktionsbasierte Identifizierung von Interaktionen und deren Bewertung über rückwirkende Prozesse anhand des M-K-I wird im Folgenden exemplarisch erörtert.

\subsection{Karstaspekt Hohlform}

Wie bereits angesprochen, stellen Hohlformen (Höhlen und Dolinen) als Ergebnis einer intensiven Lösungsverwitterung die dominierenden Endo- bzw. Exokarstformen des Untersuchungsgebiets dar.

Vor allem die in ihrer Genese horizontal ausgeprägten, zumeist trockenen Höhlen sind seit dem Paläolithikum bis in jüngste Zeit Interaktionsobjekte variierender Intensität. So belegen archäologische Fundobjekte die Nutzung der Schutzfunktion von Höhlen vor Witterung im Untersuchungsgebiet (KuSCH 1992). Trotz der beachtlichen Anzahl an Fundobjekten aus Höhlen darf nicht von einer dauerhaften Nutzung der Höhlen als Wohnsitz ausgegangen werden. Mitunter täuscht die konservatorische Wirkung von Höhlen (unzerstörte Stratigraphie) in Bezug auf das archäologische Fundmaterial (Funktion: wissenschaftlicher Wert) auch eine nicht verifizierbare starke Korrelation der Höhle-Mensch-Interaktion vor: äquivalente Murtal-nahe Freilandfunde können auch durch fluviatile Überprägungen seltener ausfallen (vgl. Fuchs 1994).

Die Interpretation von Höhlen als Kultstätten - Funktion: Orte verbergung swürdiger Aktivitäten - erweist sich als noch diffiziler. Im Untersuchungsgebiet wird diese Nutzung durch die Sekundärbestattung eines Menschen (wahrscheinlich neolithisch) in der Josefinengrotte [2836/32] vermutet (Fuchs 1994). Die genaue Zuordnung des Fundes ist durch den Verlust eines zweiten Schädels (KuSCH 1993) kaum mehr möglich.

Die Rückwirkung dieser frühen Funktionen ist anthropogene Morphodynamik im microscale (Planierung der Sedimentschichten, Eintrag allochthoner Sedimente) und mesoscale (artifizielle Veränderung des Hohlraumes). Demnach, und im Unterschied zum üblichen sozialökologischen Modell (FISCHER-KowALSKI \& HABERL 1997b), markieren Eingriffe des Menschen bereits vor der Neolithischen Revolution den Beginn von Kolonisierung natürlicher Systeme, wenngleich in geringem Ausmaß.

Ebenfalls der Funktion Orte verbergungswürdiger Aktivitäten / Produktionsstätte zugeordnet ist die Errichtung eines $\sim 2.000 \mathrm{~m}$ langen und $\sim 9.400 \mathrm{~m}^{2}$ umfassenden Stollennetzes (vgl. Abb. 6a) zur Produktion von Flugzeug-, LKW- und Panzerteilen (Typ „Tiger“) durch Konzentrationslager-Häftlinge in den Jahren 1944-45 (STELzL 1999). Das zugehörige Konzentrationslager - ein Nebenlager des KZ Mauthausen lag 2 km südlich der Stollenanlage (vgl. Abb. 5) im Ortsteil Peggau/Hinterberg. Die Größe der in nur acht Monaten errichteten Stollenanlage ist Zeugnis der Gräuel, denen die anfangs 395, später bis zu 888 Häftlinge in Zwölf-Stunden-Schichten ausgesetzt waren (FARKAS 2002). 138 aus einem Massengrab nahe des Lagers (heutiges Denkmal) exhumierte Leichen und die Exekution von 15 marschunfähigen Häftlingen vor bzw. der Tod von 20 bis 25 Häftlingen während der Auflösung des KZ und des folgenden Marsches (RABITSCH 1967) dürften nur die unterste Grenze der Opferzahlen markie- 
ren. Die tatsächliche Anzahl ist ebenso wenig bekannt wie die Identität der meisten in Massengräbern im Großraum Peggau gefundenen Leichen.

Die Rückwirkungen der artifiziellen Hohlräume (Konsequenzhöhlen) auf das Karstsystem sind neben einer macroscaligen anthropogenen Morphodynamik - 32 natürliche Hohlräume wurden im Zuge der Sprengarbeiten angefahren - auch das Aufstauen des Hammerbachs für den Antrieb einer Turbine (Änderung hydrologischer Parameter), was zu einer verstärkten Sedimentation des Quellsiphons führte (WEISSENSTEINER 1969).

Kolonisierungstätigkeit unter fortifikatorischen Aspekten führte zu artifiziellen Veränderungen zweier Höhlen bei Badl, nördlich Peggau. Beide Objekte sind Teil der geopolitisch ab 1989 obsolet gewordenen, beidseitig der Mur errichteten militärischen Talsperre als Schutz vor einem möglichen Einmarsch sowjetischer Truppen aus dem ehemaligen jugoslawischen Raum. Der Ausbau der Höhlen zu Gefechts- oder Unterständen bedingte auch einen Verschluss der Hohlräume (anthropogene Morphodynamik mesocale) durch Holz (vgl. Abb. 6b) oder Stein.

Die Eignung von Hohlformen aller Arten zur privaten Deponierung (Orte verbergungswürdiger Aktivitäten / Abfallentsorgung) gilt für jeden geomorphologischen Landschaftstyp, insbesondere aber für den hohlformenreichen Karst. Die Auffüllung von Hohlformen (Dolinen und Höhlen) als eigentliche Deponierungstätigkeit - anthropogene Morphodynamik microscale - impliziert eine mögliche, nicht intendierte Einflussnahme auf die Wasserqualität des unterlagernden Karstaquifers. Dieser wird ob seiner spezifischen Eigenschaften (u.a starke Fließgeschwindigkeit im Aquifer; gute Infiltrationsbedingungen für Schadstoffe; starke Abweichung des tatsächlichen vom orographischen Einzugsgebiet) als hoch vulnerabel attribuiert. Bei Nutzung von Karstwasser als Trinkwasser gilt es diese Faktoren sorgfältig zu analysieren. Bezüglich illegaler Deponien stellt das Untersuchungsgebiet keine Ausnahme dar: beispielhaft sei die Entsorgung im Bereich der als Ponor fungierenden Eisgrube [2836/4] genannt (vgl. Abb. 5).
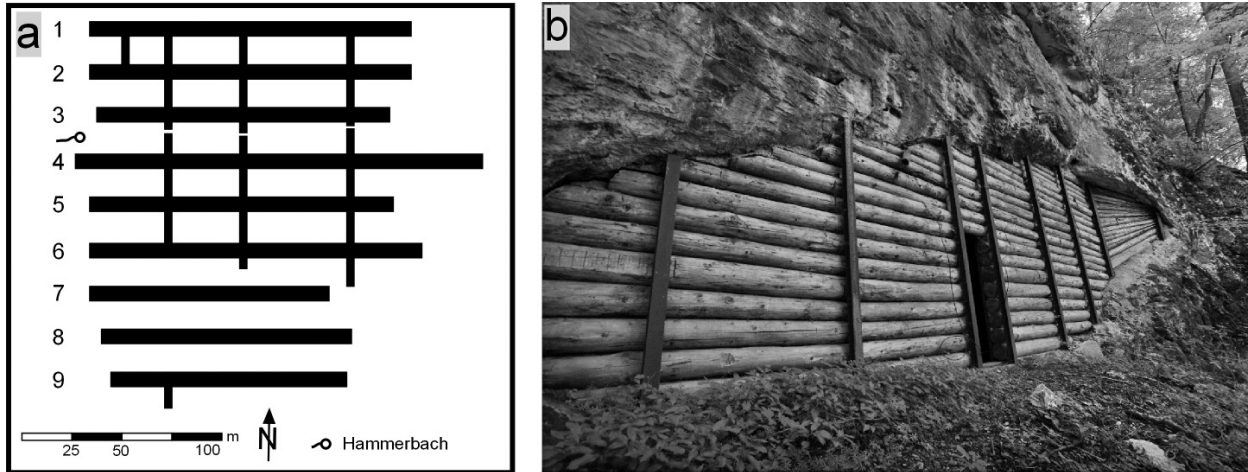

Abb. 6: Karstaspekt Hohlform

6a: Skizze der Stollenanlage des Konzentrations-Nebenlagers Peggau/Hinterberg (Lage vgl. Abb. 5). Tunnel 1-3 und 4-6 sind miteinander verbunden. Für die detaillierte HöhlenplanDarstellung [2836/34] siehe WeISSENSTEINER 1969. 6b: Bunkeranlage am rechten Ufer der Mur/ Badl. Der natürliche Hohlraum wurde mit einem Holzverbau verschlossen (Foto: C. BAUER 31.7.2008, Blickrichtung SE). 


\subsection{Karstaspekt Wasser}

Anknüpfend an das oben Genannte sei erwähnt, dass die kommunale Wasserversorgung des Untersuchungsgebietes nicht auf Karstwasser zurückgreift. Inwieweit eine Nutzung von Höhlen-Sickerwässern eine ur- und frühgeschichtliche Rolle gespielt hat, ist aufgrund sich verändernder natürlicher Rahmenbedingungen (vor allem Hydrologie) schwer rekonstruierbar, darf aber für einige Höhlen (z.B. Große Peggauer-Wand-Höhle [2836/39]) angenommen werden (KuSCH 1993).

Die Nutzung der Wässer der beiden Quellen (Hammerbach, Schmelzbach) als Energieträger - auch manifestiert in deren Namen - ist nicht karstspezifisch, wenngleich stark an karsthydrographische Eigenschaften geknüpft. So ermöglicht erst der große Gegensatz von vielen Versickerungsstellen (Einzugsgebiet des allochthonen Lurbaches; Katzenbachschwinde; Eisgrube; autochthoner Eintrag der Tannebenscholle) mit wenigen Quellen größerer Schüttung (von LeHmann 1932, S. 6 als karsthydrographischer Gegensatz postuliert) die nötige Durchflussmenge. Mit Ausnahme des bereits angesprochenen Versuchs einer Turbineninstallation im Bereich Hammerbach hat diese Nutzung keine größeren Rückwirkungen auf den Karstkörper.

$\mathrm{Zu}$ großen Kontroversen zwischen Speläologen und politischen Verantwortungsträgern führte die Installation einer Kläranlage im Semriacher Polje und die daraus resultierende Einleitung von geklärtem Abwasser in das allochthone Gewässer des Lurbaches (Wasser als Transportmedium) im Jahr 1991 (vgl. Abb. 4). Auf Seiten der Speläologen wurde die mögliche Änderung der Geschiebeführung und die Ausräumung und Verschüttung von Gangstrecken durch die erhöhte Wassermenge (per Bescheide $800 \mathrm{~m}^{3} / \mathrm{d}$ Gesamtkonsens) in einem unter Schutz gestellten Karstkörper (Naturdenkmal Tanneben, Hammerbachursprung, Lurhöhle Semriach, Doline über Dom Lurhöhle, Lurgrotte Peggau) kritisiert (TRIMmel 1994a). Demgegenüber standen Argumente der Notwendigkeit einer kommunalen Abwasserversorgung und der topographischen Ungunst (tiefe Lage im Polje). Die unterschiedlichen Nutzungsinteressen (wissenschaftlicher Wert, Naturschutz und Transportmedium) werden durch die des Schauhöhlenbetriebs Lurhöhle Semriach [2836/1a] verstärkt und führen zu einer Konkurrenz kolonisierter Systeme. Die (regelmäßige) Verschüttung der Lurbach-Schwinden im Zuge von Hochwasserereignissen bedeutet auch ein Überführen des geklärten Abwassers in den Schauhöhlenteil der Lurgrotte Semriach-Peggau (Trimmel 1994b, S. 302).

\subsection{Karstaspekt Sedimente}

Die naturräumlich bedingte inhomogene Verteilung von Ressourcen bewegt Ökonomien zum Austausch von Waren (FISCHER-KowALSKi \& HABERL 1997b). Als alternative Möglichkeiten stehen Verzicht, Adaptierung und Substitution zur Verfügung. Das folgende Beispiel zeigt Kolonisierungstätigkeiten zur Aufrechterhaltung des gesellschaftlichen Metabolismus anhand des Abbaus phosphathaltigen Materials aus Höhlen des Mittelsteirischen Karstes in den Jahren 1918 bis 1923.

Auslöser dieser Maßnahmen sind ein starker Ertragseinbruch landwirtschaftlicher Erzeugnisse (um 50-70\%) als Folge schlechter Witterung und eine unzureichende 
Menge an künstlichem Dünger im Jahr 1917 gewesen. Das während des Ersten Weltkriegs geopolitisch weitgehend isolierte Österreich konnte aufgrund einer Blockade und der Tatsache, dass viele Lagerstätten auf feindlichen Territorien lagen, keine phosphorsäurigen Düngemittel importieren (SAAR 1931). Als alternative Quellen rückten schließlich Höhlen als Phosphatlagerstätten $\left(\mathrm{P}_{2} \mathrm{O}_{5}\right)$ ins Zentrum des Interesses. Von 42 untersuchten steirischen Höhlen (davon 28 im Mittelsteirischen Karst) kam es in der Drachenhöhle bei Mixnitz [2839/1], in der Großen Badlhöhle [2837/17a] und in der Kleinen- und Großen Peggauer-Wand-Höhle III, IV, V, VI [im ÖHV: 2836/38 \& 2836/39a-d] zum Abbau (GöTZINGER 1926).

Die Phosphatlagerstätten sind organischen Ursprungs - überwiegend aus Knochen des zu den jungpleistozänen Höhlenfaunen zählenden Ursus spelaeus in Verbindung mit Lagen von Fledermausguano. Zeitlich werden primäre Lagerstätten - Höhlenbärenschichten (Knochenerden) - gegen Ende des Riss-Würm-Interstadials eingeordnet (Trimmel 1968, S. 135). Die sekundären Lagerstätten (Phosphaterden) entstanden durch Reaktion von organischem Material (Kadaver, Knochen, Guano) mit den vorhandenen klastischen Höhlensedimenten und den Höhlenwänden (FORD \& W ILLIAMS 2007, S. 293). Das größte Vorkommen lag in der Drachenhöhle bei Mixnitz - und somit außerhalb des Untersuchungsgebietes.

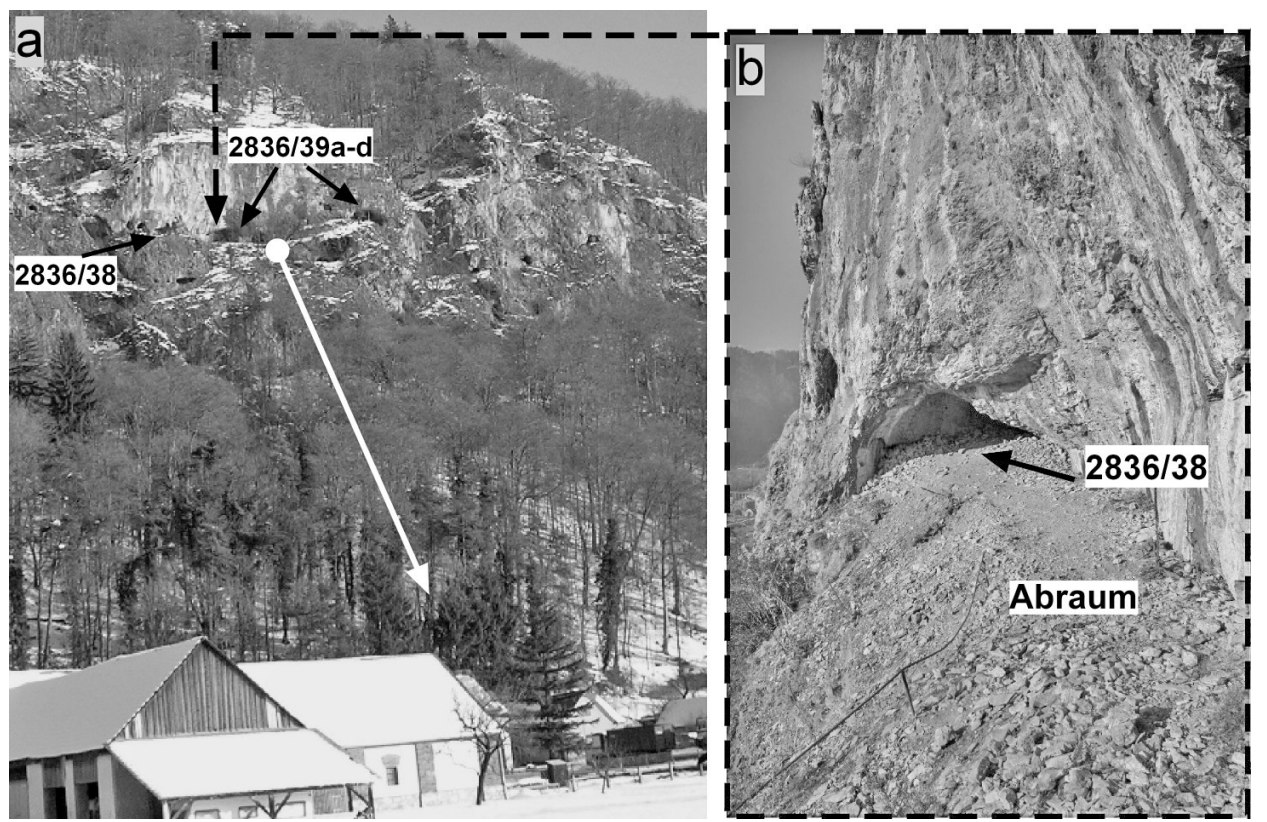

Abb. 7: Ehemaliger Phosphatabbau Peggauer Wand

7a: der weiße Pfeil markiert den ungefähren Verlauf der einstigen Seilbahnanlage (Foto: C. BAUER, 15.1.2006, Blickrichtung E). 7b: Standplatz der einstigen Förderstation am schmalen Felsband. Reste des Abraums, der auch den Höhlenmund der Kleinen Peggauer-Wand-Höhle [2836/38] fast gänzlich auffüllt, sind noch gut sichtbar (Foto: C. B AUER, 19.11.2006; Blickrichtung N). 
Der Abtransport von den schwer zugänglichen Höhlen bedeutete einen hohen logistischen Aufwand: So musste das Material der Badlhöhle über eine Holzrutsche in den Badlgraben und von dort mit einer schmalen Bahn nach Badl befördert werden. Die Lage der Peggauer-Wand-Höhlen inmitten der Felswand (140 m über der Ortschaft) erforderte die Installation einer Seilbahnanlage (vgl. Abb. 7a). Taube, sperrige Ablagerungen - sie machten im Jahr 1919 bereits 60\% des abgebauten Materials aus - wurden auf dem schmalen Felsband und in den Höhleneingängen (vgl. Abb. 7b) abgelagert. Nach nur zwei Jahren Betriebszeit erfolgte die Schließung beider Werke im Untersuchungsgebiet aus mehreren Gründen. Erstens gefährdete die zunehmend instabile Lage des Abraums am Felsband darunter liegende Gebäude. Beim Versuch des Abtransportes wurde schließlich die Förderanlage stark beschädigt (SAAR 1931). Zweitens machte die zu hoch angesetzte Schätzung von Materialmenge und $\mathrm{P}_{2} \mathrm{O}_{5}-$ Gehalt die Betriebe zunehmend defizitär. Hinzu kam eine schwankende Qualität des Produkts wegen variierendem Feuchtegehalt. Eine Qualitätssteigerung des Produktes durch Veredelung (Trocknung/Mahlen und/oder Umwandlung in Superphosphat) wurde nicht vorgenommen (Schadler 1921). Die rückwirkenden Prozesse - Ausräumung/ Umlagerung von Sedimenten und Auffüllung mit Material - betreffen größtenteils den Höhleninhalt, aber nicht die Evakuation und werden demnach der microskaligen anthropogenen Morphodynamik zugeordnet.

\section{Konkurrenz kolonisierter Systeme}

Die gegenseitige Beeinflussung kolonisierter Systeme lässt sich - als ein Effekt der großen Anzahl an Interaktionen auf sehr kleinem Areal - im Untersuchungsgebiet vielfach verifizieren. Das anschaulichste Beispiel konkurrierender kolonisierter Systeme betrifft aber jenes der Kontroversen zwischen der Funktion touristische Nutzung (Karstaspekt Formenvielfalt) und Bergbau (Karstaspekt Fläche).

Schauhöhlen sind kolonisierte Systeme mit multiplen Rückwirkungen, die im Folgenden am Beispiel der Lurgrotte Peggau [2836/1b] kurz erörtert werden. Die Attraktivität des Lurhöhlenssystems, bis zum katastrophalen Hochwasser 1975 durchgehend touristisch gangbar (vgl. Renaturierung), resultiert vorwiegend aus dessen Speläothemen und dem Einblick in eine aktive Wasserhöhle. Seit 1975 erfolgt die touristische Nutzung in Form zweier voneinander getrennter Schauhöhlen (Lurhöhle Semriach \& Lurgrotte Peggau). Erschließungsmaßnahmen am Höhlenzugang und im Höhleninneren bedeuten auch Veränderungen an der Evakuation und am Höhleninhalt. So ist der Zugang des Lurhöhlensystems von Peggau aus - einst ein wassererfüllter, unpassierbarer Siphon - erst seit 1913 durch die Anlage eines 68 m langen Entwässerungsstollens (Weissensteiner 1994) möglich. Diese Arbeiten und der Bau der notwendigen Weganlagen bedeuteten Rückwirkungen auf das Karstsystem (anthropogene Morphodynamik micro-, meso- und macroscale und Änderung hydrologischer Parameter), die auch zu Konflikten zwischen der Funktion wissenschaftlicher Wert und der Funktion touristische Nutzung führten. 

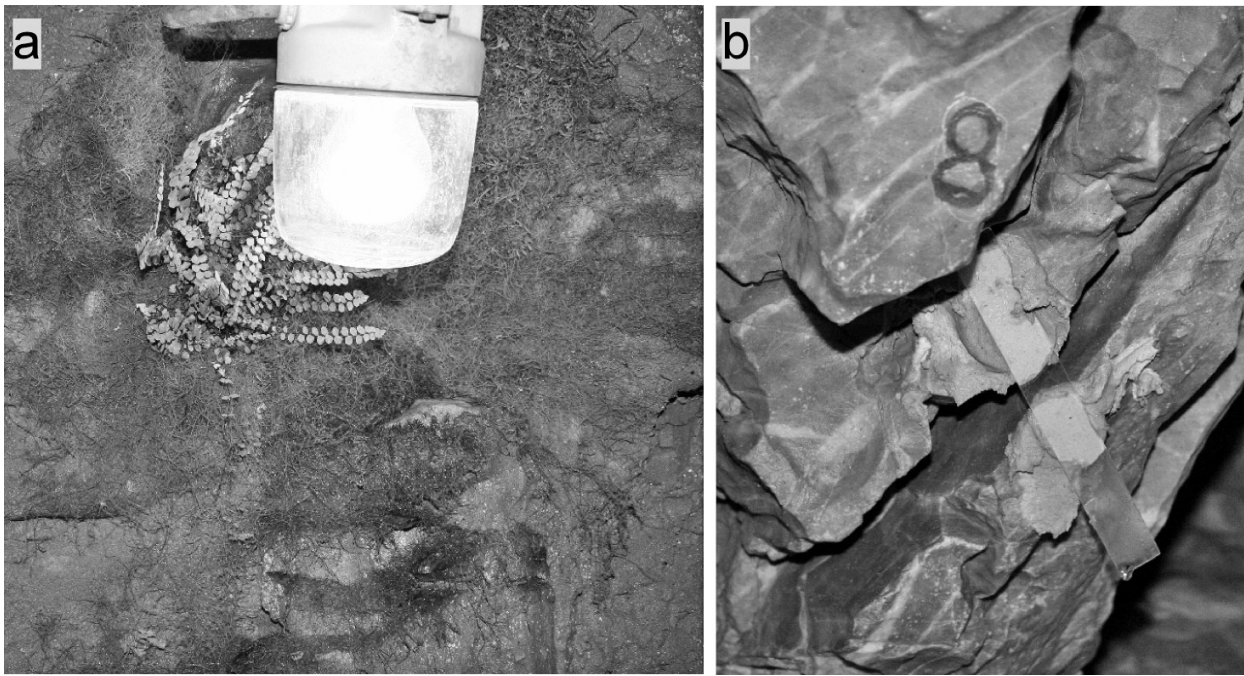

Abb. 8a: Lampenflora Lurgrotte Peggau (Foto: C. BAuer 18.3.2008). 8b: Seismisches Monitoring (Lurgrotte Peggau) möglicher Auswirkungen von Sprengungen der nahegelegenen Steinbrüche mittels sog. Spion (zwischen eine Kluft einzementierte Glasplatte oder mit Zement ausgefüllte Kluft/Fuge) (Foto: C. BAUER 18.3.2008)

Weitere, vorwiegend die Höhlenbiota betreffende Rückwirkungen ergeben sich durch den Einbau der elektrischen Beleuchtung. Der Lichtinput führt zu veränderten Lebensbedingungen für troglobionte und troglophile Faunen (vor allem Fledermäuse) und ermöglicht lichtassimilierenden Floren (Farnen, Mosen) ein Wachstum in unmittelbarer Nähe eines Beleuchtungskörpers (abhängig von der emittierten Wellenlänge). Auch wenn die Beleuchtung der Lurgrotte Peggau zeitgesteuert funktioniert und im Winter kein regulärer Führungsbetrieb stattfindet (positiv für die als Überwinterungsraum von Fledermäusen fungierende Höhle), reicht die Fotoperiode für die Etablierung einer ausgeprägten Lampenflora (vgl. Abb. 8a) aus.

Neben diesen infrastrukturell bedingten Rückwirkungen gibt es auch Effekte, die direkt von den Höhlenbesuchern initiiert werden. Unterschieden wird zwischen chemischen, biologischen und thermalen Verschmutzungen (HupPERT et al. 1993). Die Auswirkungen sind dabei vorwiegend von zwei Faktoren abhängig: erstens von den Eigenschaften der Höhle (u.a. Bewetterung, Wasserführung, Höhleninhalt) und zweitens von der Besucheranzahl. Auch wenn in Bezug auf das Lurhöhlensystem detaillierte $\mathrm{CO}_{2}$ - und Temperaturmessungen ausstehen, können doch einige grundlegende Aussagen getroffen werden. Das Lurhöhlensystem ist als dynamisch bewetterte aktive Wasserhöhle zu klassifizieren. Ohne ständige Instandhaltungstätigkeiten würden periodische Überflutungen das kolonisierte System „Schauhöhle“ schnell renaturieren. Diese Eigenschaften mindern aber auch die Gefahr der direkten Einflüsse von Touristen, wenngleich anzumerken ist, dass ein so großes Höhlensystem aus unterschiedlich ausgeprägten Passagen besteht. In Kombination mit den stagnierenden Besucherzahlen 


\begin{tabular}{|l|c|c|c|c|c|c|c|c|c|c|c|}
\hline Höhle & $\mathbf{1 9 9 7}$ & $\mathbf{1 9 9 8}$ & $\mathbf{1 9 9 9}$ & $\mathbf{2 0 0 0}$ & $\mathbf{2 0 0 1}$ & $\mathbf{2 0 0 2}$ & $\mathbf{2 0 0 3}$ & $\mathbf{2 0 0 4}$ & $\mathbf{2 0 0 5}$ & $\mathbf{2 0 0 6}$ & $\mathbf{2 0 0 7}$ \\
\hline Peggau & 21.750 & 18.930 & 17.850 & 18.960 & 16.090 & 17.590 & 17.310 & 15.710 & 16.390 & 16.430 & 17.180 \\
\hline Semriach & 19.200 & 18.630 & 19.480 & 18.280 & 17.550 & 18.430 & 18.690 & 18.120 & 20.300 & 19.900 & 20.000 \\
\hline
\end{tabular}

Quelle: mündliche Mitteilung A. Schinnerl (Lurhöhle Semriach) und G. BRAndstätter (Lurgrotte Peggau)

Tab. 4: Besucherstatistik der Lurhöhle Semriach und der Lurgrotte Peggau 1997-2007

beider Schauhöhlenbetriebe (vgl. Tab. 4) kann von einer geringen Gefahr der Übernutzung und Verschmutzung durch den Schauhöhlenbetrieb ausgegangen werden.

Der Abbau von Karbonatgestein - in der karstspezifischen Impaktforschung zu den größten "human impacts” gezählt (GunN 1993) - erfolgt derzeit im Untersuchungsgebiet an fünf Standorten, davon drei in unmittelbarer Nähe der Ortschaft Peggau (vgl. Abb. 5). Im Gegensatz zu den beiden an der Südgrenze des Untersuchungsgebiets gelegenen Steinbrüche im schwach verkarsteten Areal übt der Abbau dieser drei Standorte (zwei in Etagen und einer als Trichterabbau betrieben) in der stark verkarsteten Scholle des Tannebenstocks großen Einfluss auf den Karstkörper aus. Die Abbaumenge des Werkes Peggau stieg in den Jahren 2001 bis 2005 von 517.135 auf 654.155 t/a (WIETERSDorfer \& Peggauer Zementwerke GmbH 2006) - das entspricht einer Steigerungsrate von $\sim 26,5 \%$. Die daraus resultierende Landformenzerstörung ist in Abbildung 9a gut ersichtlich. Wie viele Karstformen im Zuge des Abbaus angeschnitten und zerstört worden sind (anthropogene Morphodynamik macroscale), ist schwer nachweisbar.

Der größte Konflikt ergibt sich aber mit der zwischen den beiden Steinbrüchen Peggau-Süd und Peggau-Nord gelegenen, als Schauhöhle genutzten Lurgrotte Peggau (vgl. Abb.9a). Auch der Trichterabbau, dessen gewonnenes Material durch einen $210 \mathrm{~m}$ tiefen Schacht mit anschließendem Förderband zum Abbau Peggau-Süd transportiert wird (vgl. Abb. 9b/c), grenzt unmittelbar an das Höhlensystem.

Die Betrachtung beider Kolonien als konkurrierende Systeme bedeutet auch ein Überführen eines Raumnutzungskonflikts in einen Raumbewertungskonflikt, dessen Priorität durch die Gesellschaft bestimmt wird. Die Konfliktsituation in Peggau erwuchs aus der im Zuge des Zweiten Weltkrieges und in der Nachkriegszeit entstandenen Nachfrage nach Baurohstoffen und der daraus resultierenden Erweiterung der Abbruchgebiete. Um ein Anfahren des Höhlensystems im Zuge des Abbruchs zu verhindern, wurden Schutzzonen definiert (KIESLInger 1957). Mögliche Auswirkungen durch Erschütterungen der für den Abbau notwendigen Sprengtätigkeiten - Großsprengungen von 30.000t/Sprengung; 15-20/a (Wietersdorfer \& PegGauer 2006) - auf den Höhleninhalt sind bis in jüngste Zeit Konfliktgegenstand. So wurden im Jahr 2000 zerstört vorgefundene Sinterröhrchen im Bereich der Höhlenpassage Drei Zinnen im Zusammenhang mit der Sprengtätigkeit gesehen (Weissensteiner 2001). Zur Dokumentation möglicher Erschütterungen im Höhlenbereich wurden sogenannte Spione installiert (vgl. Abb. 8b). Das Beispiel demonstriert eindeutig, dass eine Betrachtung von Interaktionen nur über human impacts oder disturbances (vgl. BAUER \& KELLERERPirklbauer 2009) nicht weit genug greift. Die Rückwirkungen des Schauhöhlenbetriebes - von Seiten der Wissenschaft auch vielfach artikuliert und kritisiert (TRIMMEL 1994b) - sind zweifelsfrei gegeben. Dennoch ist andererseits nicht von der Hand zu weisen, dass der Schauhöhlenbetrieb des Lurhöhlensystems seit dem Erstarken des 
Kalkbruches eine konservatorische Funktion erfüllt hat (vgl. auch ScHAFfLER 1994). Dies manifestiert sich in Renaturierungs- (nicht im sozialökologischen Sinne) und Rekultivierungsmaßnahmen im Umkreis der Steinbrüche (Abb. 9a).

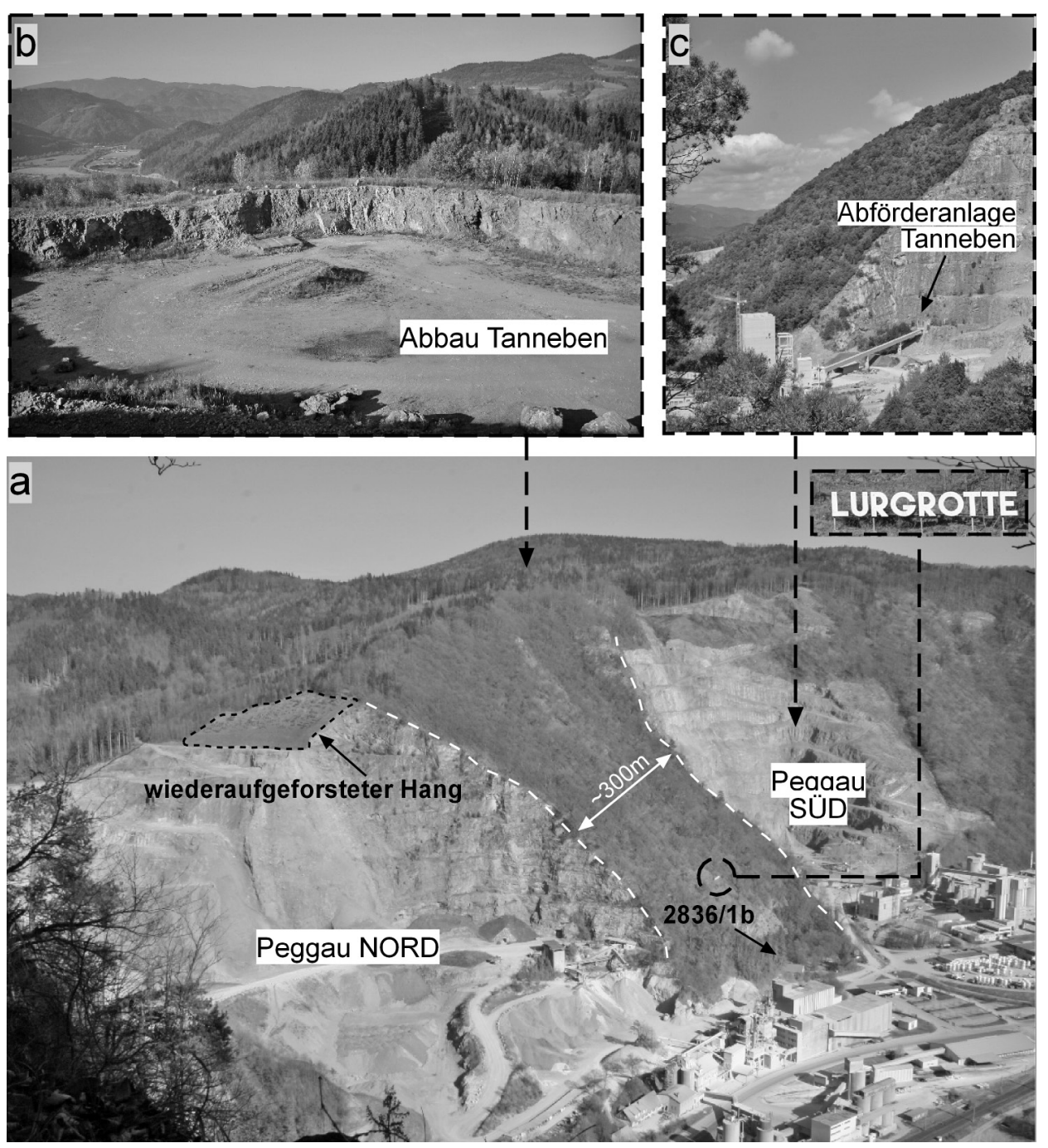

Abb. 9: Konkurrenz kolonisierter Systeme am Beispiel Peggau

9a: Kalkbruch Werk Peggau. Die weiße Linie markiert das Schutzgebiet rund um die Schauhöhle. Gut erkennbar ist die nicht mehr weiter besprengte Abbaukante im Süden des Steinbruchs Nord. Als Renaturierungs- und Kultivierungsmaßnahmen werden ehemalige Abbruchkanten abgeflacht und wieder aufgeforstet (Foto: C. BAUER 30.3.2008, Blickrichtung SE). 9b: Trichterabbau Tanneben (Foto: C. BAUER, 20.10.2008, Blickrichtung N) wurde 1992 in Betrieb genommen. 9c: Detailansicht Peggau-Süd. Erkennbar ist die Abförderanlage, die Material vom $200 \mathrm{~m}$ tiefen Schacht des Trichterabbaus Tanneben über ein $300 \mathrm{~m}$ langes Förderband zum Steinbruch Süd transportiert (Foto: C. BAUER, 11.09.2008, Blickrichtung N). 


\section{Renaturierung}

In der ökologischen Planung werden unter Rekultivierung und Renaturierung anthropogen initiierte Maßnahmen zur Rückführung eines Ökosystems in einen quasi-naturnahen Zustand verstanden (vgl. Abb. 10a). Das M-K-I versteht unter Renaturierung ein Erstarken naturaler Prozesse gegenüber anthropogenen (vgl. Abb. 10). Renaturierung ist damit zumeist kein gewünschter Prozess (kann es aber sein), sondern wirkt der Aufrechterhaltung kolonisierter Systeme (Funktionen im M-K-I) entgegen (Fischer-Kowalski \& Erb 2006).
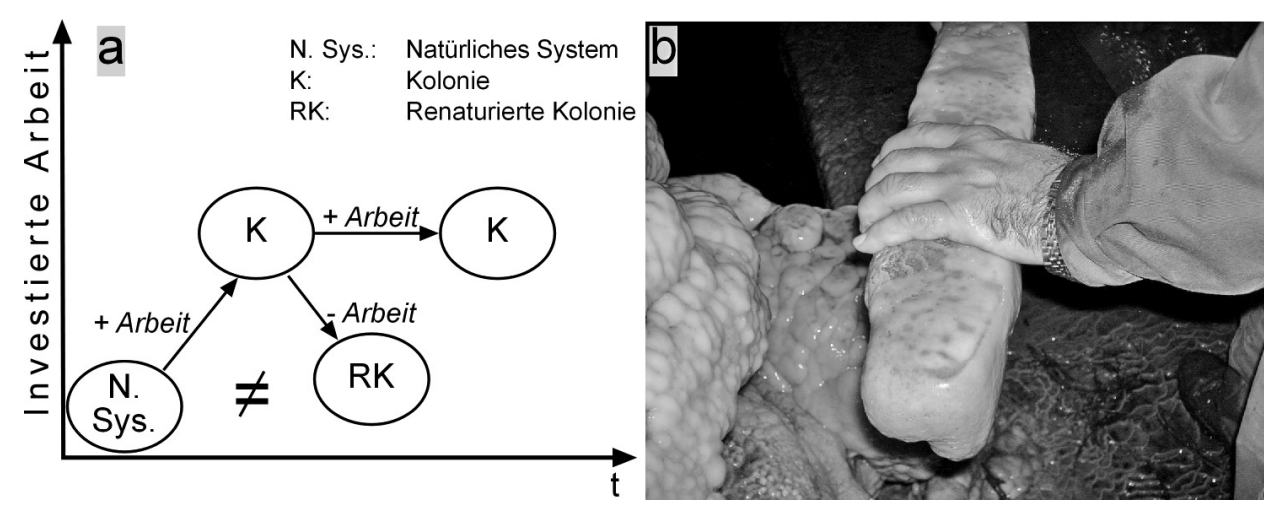

Abb. 10: Renaturierung im M-K-I

10a: Schematische Darstellung. Die Erhaltung oder Aufrechterhaltung einer Kolonie (Funktion) bedarf ständiger Investition von Arbeit. Fällt diese weg, erstarken naturale Prozesse - es entsteht eine renaturierte Kolonie. 10b: Renaturierte Kolonie am Beispiel der Lurgrotte Peggau [2836/1b]. Beschädigungen an der Schauhöhleninfrastruktur durch periodische Überschwemmungen ( jährlich) entsprechen Renaturierungen. Der Führungsweg des Mittelteils der Höhle - seit dem katastrophalen Hochwasser 1975 fast gänzlich zerstört - zeigt dies anschaulich. Gut sichtbar ist das mittlerweile stark versinterte L-Eisen des ehemaligen Geländers des Führungsweges (Foto: C. BAUER 12.11.2005).

\section{Diskussion}

Die funktionsbasierte Identifizierung von M-K-Interaktionen über das vorgestellte Modell präsentiert das Untersuchungsgebiet Tanneben (Teilgebiet 2836 des ÖHV) als vernetztes System kolonisierter Systeme. Die ersten Ergebnisse zeigen neue Facetten der Mensch-Karst-Forschung, die über die bisherigen Forschungsansätze hinausgehen oder diese erweitern, wenn wir dabei an die Anthropospeläologie (im Sinne eines den gesamten Karst umfassenden Wissenszweiges), die Angewandte Speläologie und die Historische Speläologie denken (TRImmel 1968). Als schwieriger erweist sich die angestrebte Evaluierung der kolonisierten Systeme über rückwirkende Prozesse. Erstens 
ist das Herausfiltern anthropogener Prozesse aus naturalen nicht zuletzt wegen des Einbeziehens historischer Stoffflüsse sehr schwierig. Zweitens ist die Vergleichbarkeit von Rückwirkungen im derzeit a-skalig operierenden Modell schwierig - ein Problem, das auch die Definition nominaler Skalenniveaus für alle Karstaspekte (wie in Abb. 2 für den Karstaspekt Hohlform beschrieben) nicht zufriedenstellend löst. Ähnliche (Skalen-)Probleme ergeben sich auch bei impaktorientierten Evaluierungsmodellen, wie zum Beispiel dem Karst Disturbance Index von Van Beynen und Townsend (2005). Der möglichen Kritik der Regionalisierung (das Modell wurde speziell für den Karst der Steiermark entworfen) steht dessen gute Erweiterungsmöglichkeit (z.B. um Salzwasserintrusionen, Vollformen) entgegen.

Die Beispiele der Untersuchung belegen einen massiven Einfluss durch menschliche Interaktionen, die eine Sichtweise auf menschliche Aktivität als geomorphologischen Prozess rechtfertigen (vgl. Brown 1970). Die Potenziale des Modells, vermehrt kulturwissenschaftliche Fragestellungen zu integrieren (z.B. magische Praktiken: Drachenknochen / Augennix), erfüllt die Untersuchung des Tannebenstocks allerdings nur zum Teil. Dies bleibt eventuell weiteren Untersuchungen im Karst der Steiermark vorbehalten.

\section{Dank}

Aufrichtiger Dank gebührt G.K. LieB für die Betreuung des Disserationsvorhabens und die wissenschaftliche Unterstützung, A. KelLerer-PirklbauER für die wissenschaftliche Unterstützung, C. Brandstätter (Lurgrotte Peggau), A. Schinnerl (Lurgrotte Semriach) für die Mitteilung der Besucherzahlen und schließlich G. Buchmann, C. Dolgan, W. Schweiger, und B. Wolf für die Mithilfe bei der Geländearbeit.

\section{Literaturverzeichnis}

Batsche H., Bauer F., Behrens H., Buchelta K., Hribar F., Käss W., Knuttson G., Mairhofer J., Maurin V., Moser H., Neumaier F., Ostanek L., Rrajner V., Rauert W., Sagl H., SCHNitZer W.A., ZötL J. (1967), Vergleichende Markierungsversuche im Mittelsteirischen Karst 1967. In: Steirische Beiträge z. Hydrologie, 1966/67, S. 331-404.

Bauer C., Kellerer-Pirklbauer A. (2009), Human impact on karst environments: A case study from central Styria. In: Zeitschrift f. Geomorphologie, Sondernummer (im Druck).

Benischke R., Harum T. (1994), Zur Hydrologie und Hydrogeologie des Gebietes Peggau Tanneben - Semriach. In: Landesverein für Höhlenkunde In Der Steiermark (Hrsg.), Festschrift Lurgrotte 1894-1994, S. 143-181. Graz, Styria Verlag.

Bоск H. (1913), Charakter des Mittelsteirischen Karstes. In: Mitt. f. Höhlenkunde, 6, 4, S. 5-19. Brown E.H. (1970), Man shapes the earth. In: The Geographical Journal, 136, S. 74-85.

Farkas A. (2002), Sag mir, wer die Toten sind! Personalisierung des Opfergedenkens am Beispiel der NS-Opfer von Peggau. Klagenfurt, Drava Verlag. 
Fischer-Kowalski M., ERb K. (2006), Epistemologische und konzeptionelle Grundlagen der Sozialen Ökologie. In: Mitt. d. Österr. Geogr. Ges., 148, S. 33-56.

Fischer-Kowalski M., Haberl H. (1997a), Stoffwechsel und Kolonisierung. Konzepte zur Beschreibung des Verhältnisses von Gesellschaft und Natur. In: FISCHER-KowalsKi M. (Hrsg.), Gesellschaftlicher Stoffwechsel und Kolonisierung von Natur. Ein Versuch in Sozialer Ökologie, S. 3-12. Amsterdam, Fakultas.

Fischer-Kowalski M., Haberl H. (1997b), Stoffwechsel und Kolonisierung. Ein universalhistorischer Bogen. In: FisCHER-KowALSKI M. (Hrsg.), Gesellschaftlicher Stoffwechsel und Kolonisierung von Natur. Ein Versuch in Sozialer Ökologie, S. 25-35. Amsterdam, Fakultas.

Ford D., Williams P.W. (2007), Karst Hydrogeology and Geomorphology. Chichester, Wiley. Fuchs G. (1994), Archäologie der Lurgrotte. In: LANDESverein für HöHLENKunde IN DER STEIERMARK (Hrsg.), Festschrift Lurgrotte 1894-1994, S. 85-101. Graz, Styria.

Götzinger G. (1926), Die Phosphate in Österreich. In: Mitt. d. Österr. Geogr. Ges., 69, S. 126-156.

GunN J. (1993), The geomorphological impacts of limestone quarrying. In: Williams P.W. (Hrsg.), Karst Terrains: Environmental changes and human impact (= Catena Supplement, 25), S. 187-197.

Huppert G., Burri E., Forti P., Cigna A. (1993), Effects of Tourist Development on caves and Karst. In: Williams P.W. (Hrsg.), Karst Terrains: Environmental changes and human impact (= Catena Supplement, 25), S. 251-268.

KiesLinger A. (1957), Höhlen und Steinbrüche. In: Die Höhle, 8, 4, S. 89-99.

Kusch H. (1992), Zur Stellung der Höhle in der Urgeschichtsforschung. In: Fundberichte aus Österreich, 31, S. 357-358.

Kusch H. (1993), Die kulturhistorische Bedeutung der Höhlenfundplätze entlang des mittleren Murtales (Steiermark). Univ. Graz, Diss

Kusch H. (1997), Die Grabung 1997 in der Bockhöhle bei Peggau in der Steiermark. In: Fundberichte aus Österreich, 37, S. 469-478.

Kusch H. (2004), Forschungsprojekt Moosschacht (2836/237) auf dem Tannebenstock bei Semriach, Steiermark. In: Die Höhle, 55, 1-4, S. 83-90.

Lehmann O. (1932), Die Hydrographie des Karstes. Leipzig - Wien, Franz Deuticke.

Maurin V. (1952), Ein Beitrag zur Hydrologie des Lurhöhlensystems. Die zweite Chlorierung des Lurbaches. In: Mitt. d. Naturwiss. Vereins f. Steiermark, 81/82, S. 169-180.

Maurin V. (1961), Hydrologie und Verkarstung. In: Flügel H. (Hrsg.), Die Geologie des Grazer Berglandes (= Mitt. d. Museums f. Bergbau, Geologie u. Technik am Landesmuseum Joanneum Graz, 23), S. 173-195.

Maurin V. (1994), Geologie und Karstentwicklung des Raumes Deutschfeistritz - Peggau Semriach. In: Landesverein für HöHLEnKunde in Der Steiermark (Hrsg.), Festschrift Lurgrotte 1894-1994, S. 103-137. Graz, Styria Verlag.

Paschinger H. (1965), Klimabedingte Oberflächenkarstformen am Rande der Grazer Bucht. In: Geogr. Zeitschrift, 65, S. 162-170.

RавітSсн G. (1967), Konzentrationslager in Österreich (1938-1945). Überblick und Geschehen. Univ. Wien, Diss.

SAAR R. (1931), Geschichte und Aufbau der österreichischen Höhlendüngeraktion mit besonderer Berücksichtigung des Werkes Mixnitz. In: Speläologisches Jahrbuch, 7/8, S. 3-64.

Schadler J. (1921), Die Phosphatgewinnung aus der Drachenhöhle bei Mixnitz in Steiermark. In: Berichte d. staatl. Höhlenkommission, 2, 1, S. 42-46.

SCHAFFler H. (1994), Quo vadis Tanneben? In: LANDESVEREIN FÜr HöHLENKUNDE IN DER STEIERMARK (Hrsg.), Festschrift Lurgrotte 1894-1994, S. 307-315. Graz, Styria Verlag. 
Sieferle R.P. (1997), Kulturelle Evolution des Gesellschaft-Natur-Verhältnisses. In: FischerKowALSKi M. (Hrsg.), Gesellschaftlicher Stoffwechsel und Kolonisierung von Natur. Ein Versuch in Sozialer Ökologie, S. 37-53. Amsterdam, Fakultas.

StelzL B. (1999), Lager in Graz. Zur Unterbringung ausländischer Zivilarbeiter, Kriegsgefangener und KL-Häftlinge 1938-1945. In: KarNer S. (Hrsg.), Graz in der NS-Zeit 1938-1945 (= Veröff. d. Ludwig Boltzmann-Inst. f. Kriegsfolgen-Forschungen, Sonderbd. I). Graz - Wien - Klagenfurt

Stummer G., Plan L. (2002), Handbuch zum Österreichischen Höhlenverzeichnis inklusive Bayrischer Alpenraum (= Speldok, 10).

Trimmel H. (1968), Höhlenkunde (= Die Wissenschaft. Sammlung v. Einzeldarstellungen aus allen Gebieten d. Naturwissenschaft, 126)

Trimmel H. (1994a), Das Naturdenkmal Lurhöhle (Semriach-Peggau) - Ableitungskanal für (geklärtes) Abwässer! In: Die Höhle, 45, 1, S. 1-6.

Trimmel H. (1994b), Die „Lurgrotte“ - Schauhöhlenbetrieb und Höhlenschutz. In: Landesverein für HöHLenKunde IN Der SteIermark (Hrsg.), Festschrift Lurgrotte 1894-1994, S. 293-306. Graz, Styria Verlag.

Van Beynen P., Townsend K. (2005), A Disturbance Index for Karst Environments. In: Environmental Management, 36, 1, S. 101-116.

Vormair V. (1940), Die Dolinenwelt des Mittelsteirischen Karstes. In: Zeitschrift f. Geomorphologie, 11, S. 123-150.

Weissensteiner V. (1969), Der Hammerbach bei Peggau (Steiermark, Kat.-Nr. 2836/34). In: Die Höhle, 20, 4, S. 113-123.

Weissensteiner V. (1994), Die Erforschung der Lurgrotte nach der Einschließungskatastrophe. In: Landesverein für Höhlenkunde in Der Steiermark (Hrsg.), Festschrift Lurgrotte 1894-1994, S. 53-62. Graz, Styria Verlag.

WeissensteineR V. (2001), Tätigkeitsbericht 2000 der dem Verband österreichischer Höhlenforscher angeschlossenen Vereine. In: Die Höhle, 52, 2, S. 52-54.

Wietersdorfer \& Peggauer Zementwerke GmbH (Hrsg.) (2006), Umwelterklärung Werk Peggau / Leoben. Ausgabe 2006 mit den Zahlen 2005. Klagenfurt.

WinKLER-Hermaden A. (1957), Geologisches Kräftespiel und Landformung. Grundsätzliche Erkenntnisse zur Frage junger Gebirgsbildung und Landformung. Wien, Springer Verlag. Wurmbrand G. (1871), Über die Höhlen und Grotten in dem Kalkgebirge bei Peggau. In: Mitt. d. Naturwiss. Vereins f. Steiermark, 2, 3, S. 401-427. 